\title{
Antiretrovirale Therapie der HIV-Infektion
}

\author{
Norbert H. Brockmeyer ${ }^{1}$, Bernd Salzberger ${ }^{2}$, Hans W. Doerr ${ }^{3}$, \\ Ulrich Marcus ${ }^{4}$, Hans Reinhard Brodt ${ }^{5}$
}

\section{Zusammenfassung}

Die antiretrovirale Therapie der HIV-Infektion hat sich in den letzten zwei Jahren aufgrund neuer Therapieoptionen und -strategien deutlich gewandelt. Die Empfehlungen zur antiretroviralen Therapie wurden daher in Abhängigkeit von klinischen, immunologischen und virologischen Parametern von einem deutsch-österreichischen Expertengremium überarbeitet. Eingebrachte Studienergebnisse betreffen insbesondere die Wertung der Kombinationstherapien von zwei nukleosidanalogen reverse Transkriptase-Inhibitoren (NRTI) in Kombination mit einem nichtnukleosidanalogen reverse Transkriptase-Inhibitor (NNRTI) oder einem Proteaseinhibitor und mit drei NRTI. Diese Kombinationen werden hinsichtlich ihrer therapeutischen Effektivität und

$\mathrm{D}$ ie Hemmung der Virusreplikation durch eine antiretrovirale Therapie verhindert die Krankheitsprogression, führt zur Rückbildung HIV-bedingter Symptome und zu einer klinisch relevanten Immunrekonstitution $(2,8,15,19)$.

Die zentrale pathogenetische Hypothese, die besagt, dass die fortgesetzte Virusreplikation die Progression der HIV-Infektion bestimmt, hat sich bestätigt (13). Gerade die bessere Wirksamkeit der heute verfügbaren antiretroviralen Kombinationstherapien hat jedoch die Diskussion über den idealen Zeitpunkt des Beginns einer Therapie bei einer HIV-Infektion erneut angefacht. Die Zeitspanne einer einmal begonnenen Therapie hat sich wegen der guten Wirksamkeit und der immer unwahrscheinlicher erscheinenden Möglichkeit einer Eradikation des Virus deutlich verlängert.

Für eine späte Therapieeinleitung könnte sprechen, dass die Therapie heute mit komplizierten und fehleranfälligen Einnahmevorschriften verbunden ist und Fehler bei der Einnahme zu einer Unwirksamkeit späterer Therapien führen könnten. Darüber hinaus kann die tägliche Medikamen- ihres Nebenwirkungsprofils gewichtet. Für eine effektive Therapie sind eine Resistenztestung und eine Bestimmung von Plasmamedikamentenspiegeln bedeutsame Parameter, die für das Monitoring wichtig sind, sowie Faktoren, die einen Wechsel und eine Unterbrechung der Therapie indizieren, werden aufgezeigt.

Schlüsselwörter: HIV, antiretrovirale Therapie, Resistenztestung

\section{Summary}

\section{Antiretroviral Therapy in HIV-Infections}

During the last two years, antiretroviral therapy of HIV infections has changed dramatically. Therefore a board of German and Austrian physicians experienced in antiretroviral research

teneinnahme zu einer deutlichen körperlichen und psychischen Belastung werden, insbesondere wenn sie bei asymptomatischen Patienten zu einem stärkeren Krankheitsgefühl und einer deutlichen Minderung der Lebensqualität führt. Die Langzeitnebenwirkungen der Therapie sind ebenfalls zu berücksichtigen. Außerdem ist eine klinische Besserung und Immunrekonstitution noch bei Therapiebeginn in einem weit fortgeschrittenen Stadium der HIV-Erkrankung beobachtet worden.

Für einen frühen Therapiebeginn spricht, dass Aids eine schleichend beginnende Infektionskrankheit ist, und eine antiinfektiöse Therapie generell so früh wie möglich einzuleiten ist. Zudem könnten bei lange anhaltender

\footnotetext{
$\overline{1}$ DAIG, Klinik für Dermatologie und Allergologie (Direktor: Prof. Dr. med. Peter Altmeyer) der Universität Bochum

${ }^{2}$ Klinik I für Innere Medizin (Direktor: Prof. Dr. med. Volker Diehl) der Universität zu Köln

${ }^{3}$ Institut für Medizinische Virologie (Direktor: Prof. Dr. med. Hans W. Doerr) der Universität Frankfurt/Main

${ }^{4}$ Robert Koch-Institut (Direktor: Prof. Dr. med. Reinhard Kurth), Berlin

${ }^{5}$ Medizinische Klinik III/Infektiologie (Direktor: Prof. Dr. med. Dieter Hoelzer) der Universität Frankfurt/Main
}

and HIV patient care revised the guidelines for antiretroviral therapy and treatment and have strongly been influenced by data from latest studies on clinical, immunological and viral parameters. These studies mainly investigated combination therapies such as two nucleoside reverse transcritase inhibitor (NRTI) with one protease inhibitor or one non-nucleoside reverse transcriptase

inhibitor with three NRTI. These combination therapies are evaluated in regard to their therapeutic effectiveness and their side effects. Resistance testing and drug monitoring are most important during therapy. Parameters which necessitate a change in or interruption of therapy are discussed.

Keywords: HIV, antiretroviral therapy, resist-

Replikation des HIV ein „point of no return" des Immundefekts überschritten werden oder auch durch Spontanmutationen Viren mit höherer Pathogenität entstehen.

Einigkeit besteht über das Ziel, die Progression einer asymptomatischen HIV-Infektion so lange wie möglich zu verhindern sowie darüber, eine Therapie zu beginnen, bevor irreversible Schäden des Immunsystems eingetreten sind.

Mit abnehmender Gewichtung beruhen die hier gegebenen Empfehlungen auf der Beurteilung von randomisierten kontrollierten Studien mit klinischen Endpunkten (I), randomisierten kontrollierten Studien mit Labormarkern als Endpunkten (II) und der Auswertung von weiteren klinischen pathophysiologischen und pharmakologischen Daten durch ein Expertengremium (III) (Tabelle 1). Bei den verbleibenden Unsicherheiten, insbesondere über den besten Zeitpunkt des Therapiebeginns, ist auch ein breiter Konsens mit einem möglichen Irrtum behaftet. Diese Empfehlungen wurden von einem deutsch-österreichischen Expertengremium beraten und verabschiedet. 


\section{Therapieprinzipien}

Eine Verminderung der Morbidität und Mortalität lässt sich bereits durch eine Senkung der Viruslast um circa ein bis zwei Zehnerpotenzen erreichen. Die Selektion von resistenten Virusmutanten lässt sich langanhaltend jedoch nur vermeiden, wenn die Replikation des HIV möglichst vollständig gehemmt wird. Dies erfordert eine hohe antivirale Aktivität der eingesetzten Medikamentenkombination. Die Tiefe des erreichten Nadirs (tiefster gemessener Wert) der quantitativ gemessenen HIV-RNA bestimmt wesentlich die Dauer der virologisch definierten Wirksamkeit einer Therapie. Die derzeit kommerziell erhältlichen Tests detektieren zuverlässig eine HIV-RNA-Kopienzahl von circa 50/ml Plasma. Im Vergleich zu einer Absenkung der Viruslast unter 400 HIV-Genomkopien pro ml Plasma bedeutet eine Reduzierung der Werte unter $50 / \mathrm{ml}$ mit hoher Wahrscheinlichkeit eine länger anhaltende HIV-Suppression sowie eine langsamere Resistenzentwicklung $(11,21)$. Die wichtigste Ursache für eine fehlende Replikationshemmung ist eine Resistenz des HIV gegen die entsprechenden Substanzen. Punktmutationen in den Genabschnitten, die für die reverse Transkriptase oder die virale Protease kodieren, vermitteln eine solche Resistenz (10).

Bei Indikationsstellung einer antiretroviralen Therapie muss eine Abwägung möglicher Vor- und Nachteile im Dialog zwischen spezialisiertem Arzt und gut informierten Patienten vorgenommen werden. Dies gilt vor allem für

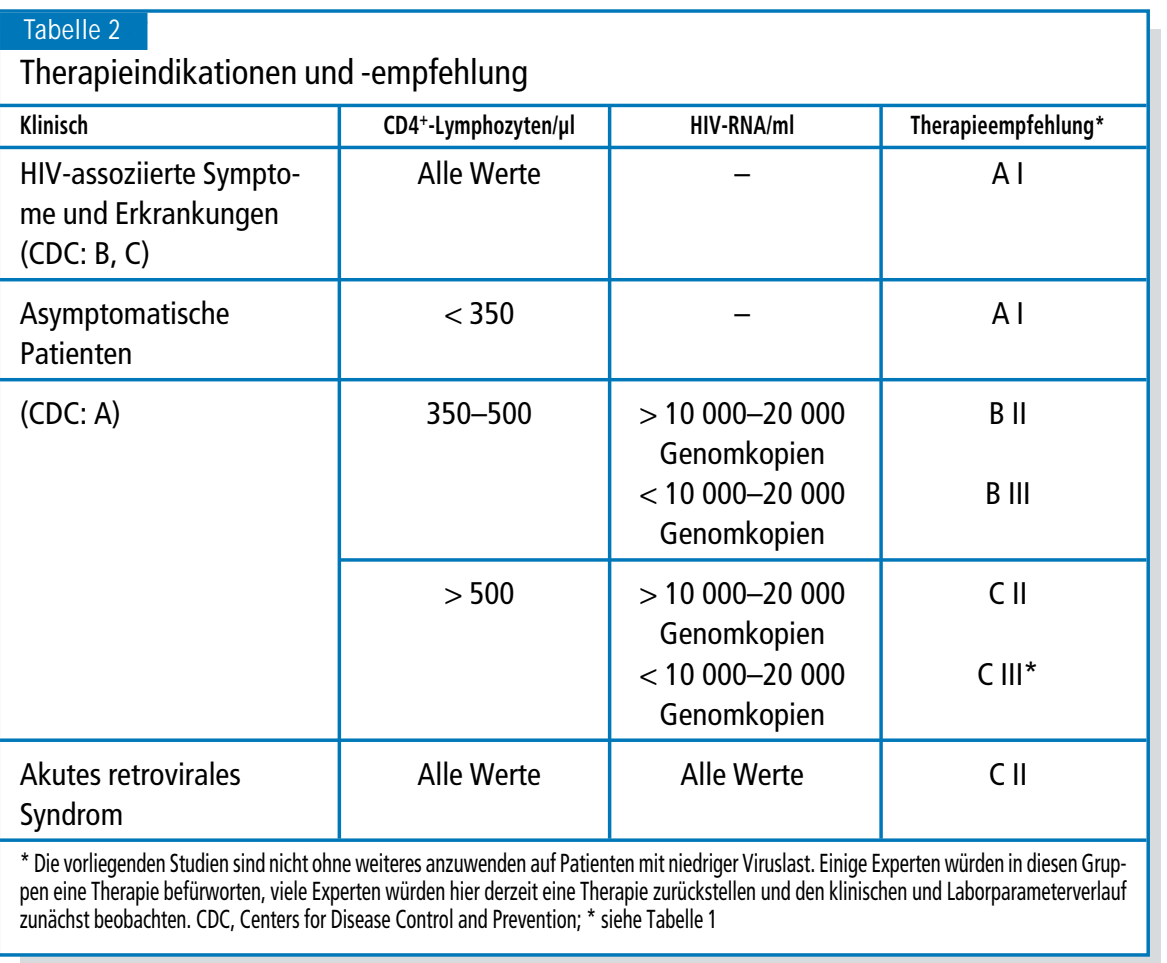

Patienten mit hohen Zahlen an $\mathrm{CD}^{+}{ }_{-}^{+}$ Zellen (Tabelle 2). Mehrere Studien zeigen, dass die vorschriftsmäßige und regelmäßige Einnahme der Medikation eine wesentliche Voraussetzung für den Erfolg einer antiretroviralen Therapie ist (5). Die dafür notwendige hohe Compliance muss in Zusammenarbeit von Arzt und Patient erreicht werden.

\section{Behandlungsindikationen}

Bisher wurde in keinem Fall gezeigt, dass ein klinischer Nachteil für antiretroviral behandelte Patienten im Vergleich zu unbehandelten Patienten in

Tabelle 1

Graduierung von Therapieempfehlungen

\begin{tabular}{|l|c|c|c|}
\hline & $\begin{array}{c}\text { I } \\
\text { Auf der Basis mindestens } \\
\text { einer randomisierten Studie } \\
\text { mit klinischen Endpunkten* }\end{array}$ & $\begin{array}{c}\text { II } \\
\text { Auf der Basis von } \\
\text { Surrogatmarker- } \\
\text { Studien }\end{array}$ & $\begin{array}{c}\text { III } \\
\text { Nach Expertenmeinung }\end{array}$ \\
\hline A Eindeutige Empfehlung & A I & A II & A III \\
\hline B Im Allgemeinen ratsam & B I & B II & B III \\
\hline C Vertretbar & C I & C II & C III \\
\hline D Im Allgemeinen abzulehnen & D I & D II & D III \\
\hline E Eindeutige Ablehnung & E I & E II & E III \\
\hline $\begin{array}{l}\text { * Klinische Endpunktstudien werden aufgrund der geänderten Zulassungsbedingungen der Federal Drug Administration (FDA) und der Europe- } \\
\text { an Agency for the Evaluation of Medicinal Products (EMEA) für neue Substanzen nicht mehr durchgeführt }\end{array}$
\end{tabular}

irgendeinem Stadium besteht. Aus diesem Grund ist für kein Stadium eine Behandlung klar abzulehnen (Empfehlung der Klasse D oder E) (Tabelle 1).

\section{Symptomatische Patienten}

Die antiretrovirale Therapie verlangsamt die Progression der HIV-Erkrankung (klinische Manifestationen $\mathrm{B}$ und $\mathrm{C}$ der klinischen Klassifikation) eindrücklich, unabhängig vom Immunstatus. Deshalb ist hier eine Behandlungsindikation gegeben und allen Patienten aus diesen Gruppen sollte eine Therapie dringend empfohlen werden (A I).

\section{Asymptomatische Patienten}

Die Grenzen der Zahl von CD4+-Lymphozyten und der HIV-Last, bei denen eine Therapie begonnen werden sollte, können nur unscharf formuliert werden. Sie liegen nach den derzeitigen Erkenntnissen bei über $350 \mathrm{CD}^{+}{ }_{-}$ Zellen/ $\mu$ l beziehungsweise zwischen 10000 bis 20000 HIV-RNA-Genomkopien/ml Plasma $(13,17,25)$.

Asymptomatische Patienten mit weniger als $350 \mathrm{CD}^{+}{ }^{+}$Zellen/ $\mu$ l Blut haben unabhängig vom Ausmaß der Vi- 
rusreplikation ein deutliches Risiko für eine immunologische und klinische Progression, das durch eine antiretrovirale Therapie vermindert werden kann $(10,11)$. Eine Behandlung für diese Patienten ist deshalb sinnvoll (A I).

Bei Patienten mit einer CD4+-Zellzahl zwischen 350 bis $500 / \mu 1$ und höherer Viruslast ( $>10000$ bis 20000 ) ist die Einleitung einer Therapie vor allem mit einer signifikanten Besserung der Surrogatmarker verbunden. Die Therapieindikation ist hier nicht eindeutig, die Therapie wird in der Regel jedoch empfohlen (B II).

Bei niedriger Viruslast $(<10000$ bis 20 000) sind Auswirkungen auf Surrogatmarker weniger deutlich und ein größerer Anteil der Experten ist zurückhaltender mit der Therapieempfehlung (B III). Patienten mit mehr als $500 \mathrm{CD}^{+}{ }^{+}$-Zellen/ $\mu$ l sollten nur behandelt werden, wenn eine deutlich messbare Virusreplikation vorliegt (>10 000 Genomkopien). Ein klinischer Vorteil für eine solche Behandlung konnte allerdings bisher nicht gezeigt werden (C II).

Die Therapieindikation für Patienten mit mehr als $500 \mathrm{CD}^{+}$-Zellen/ $\mu$, ohne oder mit nur geringer messbarer Virusreplikation, ist nicht belegt (4, 26). In diesem Stadium ist eine kurzfristige klinische Progression selten. Die Expertenmeinungen über die Indikation einer Behandlung variieren (C III).

\section{Weitere Indikationen}

Ein unbekannter Anteil von HIV-infizierten Patienten entwickelt kurz nach der Infektion und zeitnah gefolgt oder begleitet von der Serokonversion das so genannte akute retrovirale Syndrom. Es ist gekennzeichnet durch konstitutionelle Symptome, morbilliformes Exanthem, Lymphknotenschwellungen und hohe HIV-RNAWerte. Daten aus Langzeitstudien zur antiretroviralen Kombinationstherapie bei diesen Patienten liegen noch nicht vor. Studien zur Monotherapie mit Zidovudin zeigten, dass die Viruslast rasch gesenkt werden kann (12), eine Verbesserung der Langzeitprognose durch eine Monotherapie je-
Tabelle 3

Antiretrovirale Stoffklassen, Substanzen und Dosierung

\begin{tabular}{|c|c|c|c|c|}
\hline $\begin{array}{l}\text { Substanz beziehungsweise } \\
\text { Substanzgruppe }\end{array}$ & $\begin{array}{l}\text { Handels- } \\
\text { name }\end{array}$ & $\begin{array}{c}\text { Wichtigste } \\
\text { Nebenwirkungen }\end{array}$ & $\begin{array}{c}\text { Diät- } \\
\text { Vorschrift }\end{array}$ & Dosis*1 \\
\hline $\begin{array}{l}\text { Reverse Transkriptase } \\
\text { Inhibitoren - } \\
\text { Nukleosidanaloga }\end{array}$ & & $\begin{array}{c}\text { Selten } \\
\text { Laktatazidose }\end{array}$ & - & - \\
\hline Didanosin & Videx & $\begin{array}{l}\text { Pankreatitis, } \\
\text { Neuropathie }\end{array}$ & $\begin{array}{l}\text { Nüchtern } \\
\text { einnehmen }\end{array}$ & $\begin{array}{l}2 \times 200 \mathrm{mg} \\
2 \times 2 \mathrm{Tbl} .\end{array}$ \\
\hline Lamivudin & Epivir & Kopfschmerz & - & $\begin{array}{l}2 \times 150 \mathrm{mg} \\
2 \times 1 \mathrm{Tbl} .\end{array}$ \\
\hline Stavudin & Zerit & $\begin{array}{c}\text { Neuropathie, } \\
\text { Pankreatitis }\end{array}$ & - & $\begin{array}{l}2 \times 40 \mathrm{mg} \\
2 \times 1 \mathrm{Kps} .\end{array}$ \\
\hline Zalcitabin & Hivid & $\begin{array}{c}\text { Neuropathie, } \\
\text { orale Ulzera }\end{array}$ & - & $\begin{array}{c}3 \times 0,75 \mathrm{mg} \\
3 \times 1 \mathrm{Tbl} .\end{array}$ \\
\hline Zidovudin & Retrovir & Neutropenie, Anämie & - & $\begin{array}{l}2 \times 250 \mathrm{mg} \\
2 \times 1 \mathrm{Kps} .\end{array}$ \\
\hline $\begin{array}{l}\text { Lamivudin } \\
\text { Zidovudin }\end{array}$ & Combivir & $\begin{array}{c}\text { Kopfschmerz } \\
\text { Neutropenie, Anämie }\end{array}$ & - & $\begin{array}{c}2 \times 150 \mathrm{mg}+ \\
300 \mathrm{mg}, 2 \times 1 \mathrm{Tbl} .\end{array}$ \\
\hline Abacavir & Ziagen & $\begin{array}{l}\text { Hypersensitivitäts- } \\
\text { syndrom }\end{array}$ & - & $\begin{array}{l}2 \times 300 \mathrm{mg} \\
2 \times 1 \mathrm{Tbl} .\end{array}$ \\
\hline Protease-Inhibitoren ${ }^{* 2}$ & & $\begin{array}{l}\text { Glucoseintoleranz } \\
\text { Fettstoffwechselstörung } \\
\text { Lipodystrophiesyndrom } \\
\underset{* 5}{\text { Lipom }}\end{array}$ & - & \\
\hline Indinavir & Crixivan & $\begin{array}{l}\text { Nephrolithiasis, } \\
\text { Hyperbilirubinämie }\end{array}$ & $\begin{array}{l}\text { Nüchtern be- } \\
\text { ziehungsweise } \\
\text { fettreduziert } \\
\text { einnehmen }\end{array}$ & $\begin{array}{l}3 \times 800 \mathrm{mg} \\
3 \times 2 \mathrm{Kps} .\end{array}$ \\
\hline Nelfinavir & Viracept & Diarrhö, Übelkeit & $\begin{array}{l}\text { Nicht nüchtern } \\
\text { einnehmen }\end{array}$ & $\begin{array}{c}3 \times 750 \mathrm{mg} \\
3 \times 3 \mathrm{Tbl} .\end{array}$ \\
\hline Saquinavir & $\begin{array}{l}\text { Invirase }{ }^{\star 3} \\
\text { Fortovase }\end{array}$ & $\begin{array}{l}\text { Diarrhö, Übelkeit } \\
\text { (meist mild) }\end{array}$ & $\begin{array}{l}\text { Mit protein-I } \\
\text { fettreicher } \\
\text { Kost } \\
\text { einnehmen }\end{array}$ & $\begin{array}{c}2 \times 400-600 \mathrm{mg} \\
2 \times 2-3 \mathrm{Kps}{ }^{* 3} \\
3 \times 1200 \mathrm{mg} \\
3 \times 6 \mathrm{Kps} .\end{array}$ \\
\hline Ritonavir & Norvir & $\begin{array}{c}\text { Diarrhö, Übelkeit } \\
\text { Hypertriglyzeridämie }\end{array}$ & & $\begin{array}{l}2 \times 600 \mathrm{mg} \\
2 \times 7,5 \mathrm{ml}\end{array}$ \\
\hline Amprenavir & Agenerase & $\begin{array}{l}\text { Diarrhö, Kopfschmerz, } \\
\text { Arzneiexanthem }\end{array}$ & $\begin{array}{l}\text { Nüchtern be- } \\
\text { ziehungsweise } \\
\text { fettreduziert } \\
\text { einnehmen }\end{array}$ & $\begin{array}{c}2 \times 1200 \mathrm{mg} \\
2 \times 8 \mathrm{Kps} .\end{array}$ \\
\hline $\begin{array}{l}\text { Reverse Transkriptase } \\
\text { Inhibitoren - } \\
\text { nichtnukleosidisch }\end{array}$ & & Arzneireaktionen & - & - \\
\hline Nevirapin & Viramune & Arzneiexanthem & - & $\begin{array}{l}2 \times 200 \mathrm{mg} \\
2 \times 1 \mathrm{Tbl} .\end{array}$ \\
\hline Efavirenz ${ }^{* 4}$ & $\begin{array}{l}\text { Sustiva, } \\
\text { Stocrin }\end{array}$ & $\begin{array}{l}\text { Psychotrope NW; } \\
\text { Arzneiexanthem }\end{array}$ & - & $\begin{array}{l}1 \times 600 \mathrm{mg} \\
1 \times 3 \mathrm{Kps} .\end{array}$ \\
\hline Delavirdin & Rescriptor & Arzneiexanthem & - & $\begin{array}{l}3 \times 400 \mathrm{mg} \\
3 \times 4 \mathrm{Tbl} .\end{array}$ \\
\hline \multicolumn{5}{|c|}{$\begin{array}{l}\text { *1 Normale Nierenfunktion, Körpergewicht }>60 \mathrm{~kg} \\
\text { *2 Alle Proteaseinhibitoren sind Inhibitoren des Cytochrom P450, Ritonavir ist der potenteste Inhibitor, einige Isoenzyme werden durch } \\
\text { Ritonavir auch induziert } \\
\text { *3 nur in Kombination mit erprobtem weiteren PI } \\
* 4 \text { unterschiedliche Handelsnamen in Deutschland und Österreich } \\
\text { *5 Die Pathogenese des Lipodystrophiesyndroms ist noch ungeklärt. Lipodystrophiesyndrome werden auch, wenngleich seltener, bei Patienten } \\
\text { beobachtet, die nicht mit Proteaseinhibitoren, sondern mit Pl-freien Kombinationstherapien behandelt werden. }\end{array}$} \\
\hline
\end{tabular}


doch anscheinend nicht resultiert (14). Diese Befunde lassen keine Rückschlüsse auf die Therapie mit Kombinationsregimen zu. Aus diesem Grund ist eine solche Behandlung vertretbar (C II). Bezüglich der Behandlungsdauer erlauben die vorliegenden Studien keine sichere Angabe eines minimal notwendigen oder maximal sinnvollen Zeitraums. Die Behandlung dieser Patienten sollte, wenn immer möglich, im Rahmen von klinischen Studien oder standardisierten Behandlungsprogrammen geschehen, um diese offene Frage zu klären $(\mathrm{Ta}$ belle 2).

\section{Initiale Therapieregime}

Bei der Auswahl der initialen Medikamentenkombinationen sind außer Viruslast und Krankheitsstadium weitere Faktoren wie besondere Lebensweise, Komorbidität und andere notwendige Therapien zu berücksichtigen. Für eine wirksame Initialtherapie stehen folgende Optionen zur Verfügung (Tabelle 3):

- Kombination eines Proteaseinhibitors (PI) mit zwei nukleosidanalogen reverse Transkriptase-Inhibitoren (NRTI)

- Kombination eines nichtnukleosidanalogen reversen Transkriptase-Inhibitors (NNRTI) mit zwei NRTI

- Kombination von drei NRTI.

Der Stellenwert von Hydroxyurea und Adefovir in der Initialtherapie ist derzeit noch nicht geklärt. Beide Substanzen sind für diese Indikation bisher wenig untersucht und nicht zugelassen. Sie fallen deshalb aus der weiteren Betrachtung heraus.

\section{Kombinationen mit Proteaseinhibitoren}

Kombinationen aus zwei NRTI plus ein bis zwei PI führen im Vergleich zu einer stärkeren und länger anhaltenden Reduktion der Viruslast und zum stärkeren und länger anhaltenden Anstieg der $\mathrm{CD}^{+}-$Zellen, als zweifache Nukleosidanalogakombinationen. Die Wirksamkeit der PI-Kombinationen ist im Gegensatz zu den anderen Optionen auch bei Patienten mit einem weit fortgeschrittenen Immundefekt nachgewiesen worden $(9,24)$.

Nachteile der gegenwärtig verfügbaren PI sind eine ungünstige Pharmakokinetik, die die Einnahme einer großen Zahl von Tabletten in engen Zeitintervallen erfordert und die $\mathrm{Ne}$ benwirkungen und Medikamenteninteraktionen. Metabolische Störungen wie Lipodystrophie, Insulinresistenz und Diabetes werden unter PI-Kombinationen häufiger als unter anderen Kombinationen beobachtet.

\section{Kombinationen mit nichtnukleosidanalogen reversen Transkriptase-Inhibitoren}

Für NNRTI in dreifacher Kombinationstherapie liegen mittlerweile Daten einer Vergleichsstudie mit Efavirenz plus Zidovudin (AZT) plus Lamivudin (3TC) versus Indinavir plus AZT plus 3TC über eine Dauer von 48 Wochen vor. Die Kombination mit Efavirenz war in allen Analysen auch bei Patienten mit mehr als 100000 Kopi-

\section{Tabelle 4}

Basiskombinationen und Kombinationspartner

\begin{tabular}{|c|c|c|c|c|}
\hline & Nukleosidanaloga & $\begin{array}{l}\text { Therapie- } \\
\text { empfehlung*6 }\end{array}$ & $\begin{array}{l}\text { plus Proteaseinhibitor } \\
\text { oder NNRTI oder } \\
\text { dritter NRTI }\end{array}$ & $\begin{array}{l}\text { Therapie- } \\
\text { empfehlung*6 }\end{array}$ \\
\hline \multirow{12}{*}{$\begin{array}{l}\text { Empfohlene } \\
\text { Kombinationen }\end{array}$} & Zidovudin + Lamivudin & Al & Indinavir & $A \mid / / I^{* 1}$ \\
\hline & Zidovudin + Didanosin & Al & Ritonavir & $A \mid / / I^{* 1}$ \\
\hline & Zidovudin + Zalcitabin & $\mathrm{Cl}$ & Nelfinavir & All \\
\hline & Stavudin + Lamivudin & A II & Saquinavir SGC & All \\
\hline & Stavudin + Didanosin & A II & $\begin{array}{l}\text { Nelfinavir und } \\
\text { Saquinavir (SGC) }\end{array}$ & $\mathrm{CII}{ }^{* 2}$ \\
\hline & & & $\begin{array}{l}\text { Saquinavir (HGC) } \\
\text { und Ritonavir }\end{array}$ & B II \\
\hline & & & Efavirenz & All \\
\hline & & & Indinavir + Ritonavir & B II \\
\hline & Zidovudin + Lamivudin & Al & Abacavir & $\mathrm{B} \mathrm{II} * 2, * 3$ \\
\hline & Stavudin + Didanosin & A II & Nevirapin & $B \|^{* 2, * 3}$ \\
\hline & & & Delavirdin & $\mathrm{CII}{ }^{* 2, * 3}$ \\
\hline & $\begin{array}{l}\text { Stavudin + Didanosin } \\
\text { + Lamivudin }\end{array}$ & & & $C I^{* 2, * 3}$ \\
\hline \multirow{3}{*}{$\begin{array}{l}\text { Im Allgemeinen } \\
\text { abzulehnen*4 }\end{array}$} & 2 NRTI (s.o.) & & Ohne Kombination & D II \\
\hline & 2 NRTI (s.o.) & & Saquinavir HGC ein & DII \\
\hline & keine & & oder zwei PI & DII \\
\hline \multirow{3}{*}{$\begin{array}{l}\text { Eindeutig } \\
\text { abzulehnen } * 5\end{array}$} & Didanosin + Zalcitabin & E III & \multirow{3}{*}{$\begin{array}{l}\text { Jeder } \\
\text { Kombinationspartner }\end{array}$} & \\
\hline & Zidovudin + Stavudin & E II & & \\
\hline & Zalcitabin + Stavudin & E III & & \\
\hline \multicolumn{5}{|c|}{$\begin{array}{l}\text { *1 Klinische Endpunktstudien mit Indinavir und Ritonavir (Evidenzgrundlage 1) nur für Patienten mit } \mathrm{CD} 4^{+}<200 / \mu l \text {, beziehungsweise mit CD4+ } \\
<100 / \mu \text {, ansonsten Evidenz } 2 \text { für beide } \\
\text { *2 Daten nur für kurze Beobachtungsdauer vorhanden beziehungsweise noch nicht ausreichend } \\
{ }^{* 3} \text { Es liegen wenig Daten zur Therapie von Patienten mit fortgeschrittenem Immundefekt (CD4 }<350 / \mathrm{mm}^{3} \text { ) und Nukleosid-NNRTI-Kombinatio- } \\
\text { nen vor } \\
{ }^{* 4} \text { Klinisch wirksam, jedoch kurze Wirkdauer } \\
{ }^{* 5} \text { Bei den aufgeführten NRTI-Kombinationen additive Nebenwirkungen, identische Resistenzmechanismen oder kompetitive Phosphorylierung } \\
{ }^{* 6} \text { siehe Tabelle } 1 \\
\text { NNRTI, Nichtnukleosidanaloge Reverse Transkriptase-Inhibitoren; NRTI, Nukleosidanaloge reverse Transkriptase-Inhibitoren; SGC, Softgel cap- } \\
\text { sule; HGC, Hartgel capsule; Pl, Proteaseinhibitor }\end{array}$} \\
\hline
\end{tabular}


en/ml Plasma der Kombination mit Indinavir bezüglich des Anteils der Patienten mit nicht nachweisbarer HIVLast und der Verträglichkeit überlegen (22). Auch zur Kombination von Stavudin/Didanosin (d4T/ddI) und Nevirapin in der Initialtherapie zeigen Daten einer kontrollierten Studie, dass der Einsatz dieser Kombination zu ähnlichen Ergebnissen führt wie der Einsatz von d4T/ddI und Indinavir. Diese Studien wurden allerdings an Kollektiven mit nicht weit fortge-

\section{Kombinationen von drei Nukleosidanaloga}

$\mathrm{Zu}$ dreifachen NRTI-Kombinationen liegen bisher zwei Studien mit einer noch kurzen Beobachtungszeit vor. Die Aktivität und Verträglichkeit einer Kombination Abacavir/AZT/3TC (dreifach NRTI) war in einer placebokontrollierten Vergleichsstudie (über 48 Wochen) vergleichbar der dreifachen Kombination Indinavir/Combivir (23). Die Kombination Didano-

Tabelle 5

Interaktionen: Proteaseinhibitoren + Nichtnukleosidanaloge reverse TranskriptaseInhibitoren

\begin{tabular}{|c|c|c|c|c|c|c|c|}
\hline & $\begin{array}{l}\text { Indinavir } \\
\text { (IDV) }\end{array}$ & \begin{tabular}{|l}
$\begin{array}{l}\text { Ritonavir } \\
\text { (RTV) }\end{array}$ \\
\end{tabular} & \begin{tabular}{|l} 
Saquinavir \\
(SQV)
\end{tabular} & $\begin{array}{l}\text { Nelfinavir } \\
\text { (NFV) }\end{array}$ & \begin{tabular}{|l} 
Nevirapin \\
(NVP)
\end{tabular} & \begin{tabular}{|l} 
Delavirdin \\
(DLV)
\end{tabular} & $\begin{array}{l}\text { Efavirenz } \\
\text { (EFV) }\end{array}$ \\
\hline IDV & & $\begin{array}{l}\text { IDV 5x } \uparrow \\
\text { RTV } \leftrightarrow\end{array}$ & $\begin{array}{l}\text { IDV } \leftrightarrow \\
\text { SQV 4-7x }\end{array}$ & $\begin{array}{l}\text { IDV } 50 \% \uparrow \\
\text { NFV } 80 \% \uparrow\end{array}$ & $\begin{array}{l}\text { IDV } 28 \% \uparrow \\
\text { NVP↔ }\end{array}$ & $\begin{array}{l}\text { IDV } 40 \% \uparrow \\
\text { DLV } \leftrightarrow\end{array}$ & $\begin{array}{l}\text { IDV } 30- \\
35 \% \downarrow\end{array}$ \\
\hline RTV & & & $\begin{array}{l}\text { RTV } \leftrightarrow \\
\text { SQV 20x } \uparrow\end{array}$ & $\begin{array}{l}\text { RTV } \leftrightarrow \\
\text { NFV } 1,5 x \uparrow\end{array}$ & RTV $15 \% \downarrow$ & RTV $70 \% \uparrow$ & $\begin{array}{l}\text { RTV } 20 \% \uparrow \\
\text { EFV } 20 \% \uparrow\end{array}$ \\
\hline SQV & & & & $\begin{array}{l}\text { SQV } 3-5 x \uparrow \\
\text { NFV } 20 \% \uparrow\end{array}$ & $\begin{array}{l}\text { SQV } 25 \% \downarrow \\
\text { NVP }<5 \% \downarrow\end{array}$ & SQV 5x $\uparrow$ & $\begin{array}{l}\text { SQV } 60 \% \downarrow \\
\text { EFV } 10 \% \downarrow\end{array}$ \\
\hline NFV & & & & & $\begin{array}{l}\text { NVP } 10 \% \downarrow \\
50 \% \uparrow \\
\text { NFV } 10 \% \uparrow\end{array}$ & $\begin{array}{l}\text { NFV } 2 x \uparrow \\
\text { DLV } 50 \% \downarrow\end{array}$ & NFV $26 \% \uparrow$ \\
\hline NVP & & & & & & keine Daten & keine Daten \\
\hline DLV & & & & & & & keine Daten \\
\hline EFV & & & & & & & \\
\hline
\end{tabular}

PI, Proteaseinhibitor; NNRTI, Nichtnukleosidaler reverse Transkriptase-Inhibitor; $\uparrow$, Serumkonzentrationserhöhung; $\leftrightarrow$, keine Wechselwirkung; $\downarrow$, Serumkonzentrationsabnahme

schrittenem Immundefekt durchgeführt und können vorläufig nicht auf Patienten mit weit fortgeschrittenem Immundefekt (zum Beispiel $\mathrm{CD}^{+}{ }^{+}$ Zellzahl < 100/ $\mu$ l) übertragen werden. Hierzu sind weitere Daten zur Effektivität erforderlich.

Vorteile der NNRTI-Kombinationen sind bessere Pharmakokinetik (Nevirapin wird zweimal täglich, Efavirenz einmal täglich gegeben) und geringere Zahl von Tabletten. Efavirenz und Nevirapin werden ebenfalls über das Cytochrom-P450-System metabolisiert, Interaktionen mit anderen Medikamenten sind aus diesem Grund auch hier vorhanden, allerdings nicht so ausgeprägt wie bei Proteaseinhibitoren (18). $\sin /$ Stavudin/Lamivudin war in einer offenen randomisierten Studie in ihrer antiviralen Wirksamkeit bei niedriger Viruslast vergleichbar den Kombinationen Didanosin/Stavudin/Crixivan und Didanosin/Stavudin/Nevirapin. Vorteile der dreifachen NRTI-Kombination sind die einfache Dosierung (minimal zweimal täglich zwei Kapseln) und geringe Interaktionen mit anderen Therapeutika (18).

\section{Bewertung}

Die ersten beiden Therapieoptionen sind bezüglich ihrer antiretroviralen Wirksamkeit vergleichbar. Die Immunrekonstitution bei Patienten in weit fortgeschrittenem Krankheitsstadium wurde unter PI-freien Medikamentenkombinationen bisher nicht in gleichem Ausmaß belegt. Unterschiede im Nebenwirkungsspektrum sind vorhanden.

Als klinisch additiv wirksam mit Nukleosidanalogakombination haben sich bisher nur die Proteaseinhibitoren Indinavir, Ritonavir und Saquinavir erwiesen, allerdings nur für Patienten mit einer Zellzahl unter $350 \mathrm{CD}^{+}{ }_{-}$ Zellen $/ \mathrm{mm}^{3}$. Aufgrund dieser Daten sollte bei diesen Patienten mit weit fortgeschrittenem Immundefekt bei Therapiebeginn, wenn möglich, ein Proteaseinhibitor Bestandteil der initialen Kombination sein. Dreifache Nukleosidanalogakombinationen sollten aufgrund der noch kurzen Laufzeit der entsprechenden Studien nur als Alternative eingesetzt werden, wenn eine Therapie mit Proteaseinhibitoren oder NNRTI nicht durchführbar ist (17) (Tabelle 4).

\section{Verlaufskontrollen und Monitoring}

Die wichtigsten Laborparameter für die Verlaufsbeurteilung einer HIV-Infektion sind die quantitative Bestimmung der $\mathrm{CD} 4^{+}$-Lymphozyten und der HIV-RNA. Sie sollten zum Zeitpunkt der Diagnosestellung und anschlieBend in circa zwei- bis dreimonatigen Abständen bestimmt werden, und zwar mit dem jeweils sensitivsten erhältlichen Test. Die Einleitung und Umstellungen einer Therapie sind Indikationen für kurzfristigere Kontrollen.

Bei Patienten unter Therapie, deren HIV-RNA unterhalb der Nachweisgrenze (zurzeit 50 Genomkopien/ml) liegt, sollte die Viruslast circa alle zwei bis drei Monate kontrolliert werden. Eine signifikante Veränderung der Virusreplikation ist ab einer Änderung von 0,5 bis 0,7 Zehnerpotenzen (entsprechend Veränderungen um den Faktor drei bis sechs) anzunehmen. Signifikante Veränderungen der $\mathrm{CD}^{+}{ }^{+}-$Werte sind ab einem Abfall von 30 Prozent für Absolutwerte oder um drei Prozent für Relativwerte anzunehmen. Insbesondere Messungen, die Anlass zu einer Neubewertung der 
Therapie geben, sollten kurzfristig wiederholt werden. In der Regel sind jedoch Messungen im Abstand von weniger als vier Wochen nicht notwendig.

\section{Therapieerfolg und -versagen}

Ein Therapieerfolg kann frühestens nach vier Wochen, oft erst nach drei Monaten und in Einzelfällen erst nach sechs Monaten beurteilt werden. Ein Absinken der HIV-Replikation unter die Nachweisgrenze ist als Therapieerfolg zu werten. Ein geringerer Abfall der HIV-RNA (eine Zehnerpotenz) nach vier Wochen oder das Ausbleiben des Abfalls unter die Nachweisgrenze innerhalb von maximal sechs Monaten, ist ein ungenügender Therapieerfolg und sollte Anlass sein, additive oder alternative Therapieregime zu erwägen.

Eine relevante Einbuße der Wirksamkeit liegt wahrscheinlich vor, wenn die HIV-RNA über den Nadir des Abfalls ansteigt. Von einem sekundären Versagen der Therapie ist auszugehen, wenn die HIV-RNA wieder auf einen Wert ansteigt, der nur noch eine Zehnerpotenz unterhalb des Ausgangswerts liegt.

Hinweise auf eine ungenügende Wirksamkeit sind ferner ein signifikanter Abfall der CD4+-Lymphozyten sowie eine weitere klinische Progression. Insbesondere ein Therapieversagen nach dem letzten Kriterium zu bewerten ist oft nicht einfach. Eine antiretrovirale Therapie kann virologisch wirksam, das Immunsystem aber bereits so schwer geschädigt sein, dass trotzdem eine opportunistische Erkrankung auftritt. Auch kann die Immunrekonstitution durch eine begonnene antiretrovirale Therapie zur Exazerbation von Erkrankungen führen (so genannte Immunrekonstitutionssyndrom).

\section{Resistenztestung}

Bei einem Teil der Patienten kommt es unter Therapie zu einem Wiederanstieg der Viruslast. Eine der Hauptursachen für das Therapieversagen ist eine Resistenzentwicklung gegen die eingesetzten Medikamente. Ein sinn- voller Wechsel der antiretroviralen Therapie in dieser Situation ist ohne Kenntnis der Virusresistenz schwierig. Zum einen ist die Zahl der möglichen sinnvollen Medikamentenkombinationen deutlich kleiner als die der auf dem Markt befindlichen Präparate, zum anderen sind für eine Reihe von Medikamenten Kreuzresistenzen beschrieben.

Zur Bestimmung der Resistenz sind genotypische und phänotypische Verfahren etabliert, die sich gegenseitig ergänzen. Der Nachweis entsprechender Resistenzmutationen auf der einen Seite und die funktionelle Testung der Virushemmung durch die antiretroviralen Substanzen auf der anderen Seite erlauben eine detaillierte $\mathrm{Er}$ mittlung wirksamer beziehungsweise unwirksamer Medikamente und somit eine gezielte Therapie $(1,3,6,16)$.

Während die Durchführung einer HIV-Resistenztestung vor dem Beginn einer antiretroviralen Therapie aufgrund des derzeitigen Wissenstands noch nicht als zwingend notwendig angesehen wird (C III), gibt es Konstellationen, in denen eine Resistenztestung durchgeführt werden sollte. Dies ist der Fall vor einem notwendigen Therapiewechsel aufgrund von Unwirksamkeit des bisherigen therapeutischen Regimes A II sowie bei Verdacht auf HIV-Infektion mit einem primär resistenten Virus (zum Beispiel bei einem antiretroviral vorbehandelten Patienten als vermuteter Infektionsquelle) (B III).

\section{Serumspiegelbestimmung}

Bei Versagen einer antiretroviralen Therapie sollte immer auch eine mangelnde Compliance oder Bioverfügbarkeit als möglicher Grund erwogen und ausgeschlossen werden. Serumkonzentrationen der PI und NNRTI weisen eine klare Korrelation mit Wirkungen und Nebenwirkungen auf. Serumspiegelkontrollen von NRTI sind nicht sinnvoll. Die Bestimmung von Serumspiegeln von PI und NNRTI können vor allem bei Hinweisen auf Absorptionsstörungen und bei komplexen Wirkstoffkombinationen, die zu Interaktionen führen können, sinnvoll sein (Tabelle 5).

\section{Therapiewechsel und -unterbrechung}

Änderungen der Therapie können aufgrund von Unwirksamkeit und Nebenwirkungen notwendig werden. Eine klare Definition eines Versagens einer antiretroviralen Therapie kann derzeit nicht gegeben werden. Eine Reihe von Experten sehen jeden kontrollierten Wiederanstieg der HIV-RNA vom nicht messbaren in den messbaren $\mathrm{Be}$ reich als Versagen an. Die konservativste Definition geht von einem Wiederanstieg in den Bereich von weniger als einer Zehnerpotenz unterhalb des Ausgangswerts aus. Das bei einem Therapieversagen auszuwählende Alternativregime sollte einen Wechsel möglichst aller Substanzen beinhalten sowie den Einsatz einer neuen Substanzklasse. Nicht entschieden werden kann derzeit, ob eine Fortsetzung einer virologisch unwirksamen Therapie einen Vorteil darstellt im Vergleich zum Absetzen, falls keine Möglichkeit zum sinnvollen Wechsel mehr besteht. Insbesondere Entscheidungen über Zweit- und Alternativtherapien erfordern Spezialkenntnisse und sollten nur von besonders erfahrenen und informierten Ärzten getroffen werden.

Eine Umstellung einer wirksamen Therapie bei Patienten mit schweren Nebenwirkungen ist selbstverständlich möglich. Dies ist die einzige klinische Situation, in der zum Austausch nur eines Medikaments geraten werden kann. Bei notwendigen Therapieunterbrechungen sind alle Substanzen gleichzeitig (gegebenenfalls unter Beachtung der pharmakologischen Daten) abzusetzen (B III). Unterbrechungen oder Pausen der antiretroviralen Therapie werden derzeit in mehreren Studien auf ihre Langzeitwirkung untersucht. Pausen außerhalb von Therapiestudien können nicht empfohlen werden.

\section{Schwangere und Kinder}

Es liegen Empfehlungen zur antiretroviralen Therapie bei HIV-infizierten Kindern vor (27). Zur Therapie in der Schwangerschaft und zur Postexpositionsprophylaxe nach HIV-Exposition sind deutsch-österreichische Empfehlungen verfasst worden $(7,20)$. 
Die vorstehende Text wurde erarbeitet im Auftrage der Deutschen Aids-Gesellschaft (DAIG) und der Österreichischen Aids-Gesellschaft (ÖAG) sowie der Arzneimittelkommission der deutschen Ärzteschaft (AkdÄ), der Deutschen Arbeitsgemeinschaft niedergelassener Ärzte in der Versorgung von HIV- und Aids-Patienten (DAGNÄ), der Deutschen Cochrane-Gruppe, der Deutschen Gesellschaft für Chirurgie, der Deutschen Dermatologischen Gesellschaft (DDG), der Deutschen Gesellschaft für Infektiologie (DGI), der Deutschen Gesellschaft für Innere Medizin (DGIM), der Deutschen Gesellschaft für Krankenhaushygiene (DGKH), der Deutschen Gesellschaft für Mund-, Kiefer- und Gesichtschirurgie (DGMKG), der Deutschen Gesellschaft für Pneumologie (DGP), der Deutschen STD-Gesellschaft (DSTDG), der Deutschen Gesellschaft für Transfusionsmedizin und Immunhämatologie (DGTI), der Deutschen Vereinigung zur Bekämpfung der Viruskrankheiten (DVV) und der Kommission für Antivirale Chemotherapie der Gesellschaft für Virologie (GfV), der Deutschen Aids-Hilfe (DAH), Projekt Information, des Nationalen Referenzzentrums für Retroviren (NRZ), Erlangen und des Robert Koch-Instituts (RKI) unter der Mitarbeit von: C. Ambrecht, Düsseldorf, K. Arastèh, Berlin, A. Bader, Stuttgart, T. Berg, Potsdam, J. Bogner, München, W. Brockhaus, Nürnberg, N.H. Brockmeyer, Bochum, H.R. Brodt, Frankfurt (M.), H. Busch, Münster, H.W. Doerr, Frankfurt (M.), S. Dupke, Berlin, L. Ebert, Berlin, S. Esser, Essen, B. Fleckenstein, Erlangen, F.-D. Goebel, München, J. Gölz, Berlin, I. Grosch-Wörner, Berlin, L. Gürtler, Greifswald, M. Hartmann, Heidelberg, E.-B. Helm, Frankfurt, H. Jäger, München, H. Jablonowski, Salzgitter, J. Jarke, Hamburg, P. Kern, Ulm, H. Knechten, Aachen, T. Körner, Hannover, K. Korn, Erlangen, A. Kramer, Greifswald, R. H. Kriegel, Berlin, M. Kurowski, Berlin, M. L'age, Berlin, J. v. Lunzen, Hamburg, U. Marcus, Berlin,T. Mertens, Ulm, V. Miller, Frankfurt, A. Moll, Berlin, B. Pfeil, Leipzig, A. Plettenberg, Hamburg, H. Rasokat, Köln, T. Remè, Hamburg, A. Rieger, Wien, J. Rockstroh, Bonn, H. Rübsamen- Waigmann, Wuppertal, B. Ruf, Leipzig, J.A. Rump, Freiburg, A. Sakrauski, München, B. Salzberger, Köln, T. Schaberg, Rotenburg, I. Schedel, Hannover, B. Schmied, Wien, B. Schmidt, Erlangen, R.E. Schmidt, Hannover, H.-H.. Schmitz, Hamburg, H. Schöfer, Frankfurt (M.), M. Schrappe, Köln, S. Staszewski, Frankfurt (M.), I. Steidl, München,H.-J. Stellbrink, Hamburg, A. Stoehr, Hamburg, M. Stoll, Hannover, E. Tschachler, Wien, N. Vetter, Wien, B. Vielhaber, Berlin, A. Werner, Langen, R. Zangerle, Innsbruck.

Zitierweise dieses Beitrags:

Dt Ärztebl 2001; 98: A 175-181 [Heft 4]

Die Zahlen in Klammern beziehen sich auf das Literaturverzeichnis, das über den Sonderdruck beim Verfasser und über das Internet (www.aerzteblatt.de) erhältlich ist.

Anschrift für die Verfasser:

Prof. Dr. med. Norbert H. Brockmeyer

Deutsche Aids-Gesellschaft (DAIG)

Dermatologische und Allergologische Klinik der Ruhr-Universität

Gudrunstraße 56, 44791 Bochum

\section{Referiert}

\section{H. pylori produziert endogenes Antazidum}

In mehreren Studien konnte gezeigt werden, dass die Wirkung von Protonenpumpenhemmern (PPI) nach einer Helicobacter-pylori-Sanierung der Magenschleimhaut nachlässt. Die Autoren führten Magensekretionsstudien vor und ein sowie sechs Monate nach Eradikation einer Helicobacter-pylori-Infektion durch, wobei das Ammoniak, die Gallensäuren und die Eiweißkonzentration im Magensaft festgehalten wurden. Der höhere $\mathrm{pH}$-Wert des Magens, der vor Sanierung der Infektion beobachtet wurde, geht zum Großteil auf die Ammoniak- produktion zurück, wobei die PPI-Therapie und der duodenogastrale Reflux keine Rolle spielen. Offensichtlich produziert Helicobacter pylori ein ,endogenes Antazidum", das etwa 50 Prozent der innerhalb von 24 Stunden produzierten Säuremenge zu puffern vermag.

Bercik $P$, Verdú E F, Armstrong $D$ et al.: The effect of ammonia on omeprazole-induced reduction of gastric acidity in subjects with helicobacter pylori infection. Am J Gastroenterol 2000; 95: 947-955.

Premysil Bercik, M.D., Intestinal Diseases Research Programme, McMaster University, 1200 Main Street West, Hamilton, Ontario L8N 3Z5, Kanada.

\section{Referiert}

\section{Zidovudin zur Prophylaxe der perinatalen HIV-Übertragung}

An einer großen Zahl HIV-infizierter Schwangerer wurden kurze und lange Therapieschemata mit Zidovudin zur Prävention einer materno-fetalen HIVÜbertragung in der Perinatalphase untersucht. Dabei zeigte sich die Kombination aus langdauernder Therapie der Mutter (28. Schwangerschaftswoche bis zur Geburt) kombiniert mit langdauernder Therapie der Neugeborenen (sechs Wochen ab Geburt) allen anderen Therapieschemata mit verkürzten Zidovudin-Gaben sowohl bei der Mutter als

\section{Referiert}

\section{Krebsrisiko bei perniziöser Anämie}

Bei Patienten mit chronisch atrophischer Gastritis und intestinaler Metaplasie auf dem Boden einer Autoimmungastritis, wie dies für eine perniziöse Anämie kennzeichnend ist, entwickeln sich gehäuft Magenkarzinome. Aber auch eine Assoziation mit dem Pankreas- und Ösophaguskarzinom ist in der Literatur aufgeführt. Die Autoren berichten über die Ergebnisse einer Untersuchung an 4586 Patienten mit perniziöser Anämie, die über das schwedische Krebsregister erfasst worden waren. Sie konnten nur einen Zusammenhang mit dem Magenkar- auch beim Neugeborenen überlegen. Von besonderer Bedeutung erwies sich die lange Behandlungsdauer der Mütter. Die Autoren empfehlen daher, diese in jedem Fall durchzuführen, da längere Therapiezeiten bei den Neugeborenen Versäumnisse in der Pränatalphase nicht mehr wettmachen können.

acc

Lallemant $\mathrm{M}$ et al.: A trial of shortened zidovudine regimens to prevent mother-to-child transmission of human immunodeficiency virus type 1. N Eng J Med 2000; 343: 982-991.

Dr. Lallemant, PHPT, 57/2 Faham Rd., Soi 3, Muang Chiang Mai 50000, Thailand. zinom, nicht jedoch mit einem Karzinom von Speiseröhre und Bauchspeicheldrüse dokumentieren. Insgesamt wurden 54 Fälle eines Magenkarzinoms registriert, wobei sowohl der chronische Entzündungsprozess in der Magenschleimhaut als auch die perniziöse Anämie als Risikofaktoren anzusehen sind.

Karlsin BM, Ekbom A, Wacholder S et al.: Cancer of the upper gastrointestinal tract among patients with pernicious anemia: a case-chort study. Scand J Gastroenterol 2000; 35: 847-851.

Dr. B. M. Karlson, Department of Surgery, University Hospital, SE-751 85 Uppsala, Schweden. 